\title{
Arbor
}

\section{La conservación de los tejidos}

\author{
$M^{a}$ Socorro Mantilla de los Ríos Rojas, Mónica Moreno \\ García
}

Arbor CLXIX, 667-668 (Julio-Agosto 2001), 677-690 pp.

Entendiendo los tejidos antiguos como verdaderas obras de arte, olvidando el concepto de que estos pertenecen a las llamadas «artes menores», consideremos la amplia problemática que supone asegurar su conservación.

Los tejidos, como tal materia orgánica, se encuentran a la cabeza de los materiales más vulnerables dentro del conjunto de las obras de arte, hecho que viene motivado por su propia naturaleza extremadamente sensible a los agentes externos.

Según se trate del origen de las fibras que constituyen los tejidos estableceremos la división en dos grandes grupos que se comportan de forma diferente ante las mismas condiciones ambientales. Se trata de los tejidos constituidos por fibras proteínicas y los formados por fibras celulósicas.

La conservación de los tejidos antiguos queremos considerarla bajo dos aspectos fundamentales: la conservación en su sentido más amplio, como factor independiente de la restauración, que supone la intervención directa sobre la obra de arte.

Para poder determinar el estado de conservación de los materiales textiles es de gran importancia conocer su naturaleza, para lo cual recurrimos a laboratorios de los centros que hoy día disponen de técnicas analíticas puestas a punto y que nos suministrarán los datos necesarios.

Como técnicas de identificación a las que se puede recurrir de manera más asequible contamos con la microscopia en sus dos vertientes, óptica y electrónica. La microscopia óptica pondrá de manifiesto las características de las superficies y de las secciones transversales de las fibras. Que nos dará los datos complementarios para la total identificación de la materia que estudiamos. 


\section{Técnicas de identificación}

Solo recurriremos a la microscopia electrónica en casos muy concretos y solo si disponemos de análisis comparativos que nos dará el tipo de alteración o el agente que produjo esta.

En las colecciones de los museos es muy frecuente encontrar piezas textiles teñidas en épocas muy antiguas en las que solo se tenía conocimiento de tintes de origen vegetal o animal, y otros de origen más reciente en los que se han utilizado colorantes de sintesis conocidos como tintes artificiales.

Como norma general para obtener los datos identificativos de estas substancias recurriremos de nuevo a los centros mencionados anteriormente.

La técnica nalítica por excelencia para la identificación de los colorantes es la cromatografía tanto de capa fina como de alta resolución, siendo más completa la segunda por aportarnos datos cualitativos y cuantitativos de las materias utilizadas en la tincción del tejido.

Si conocemos el colorante con el que ha sido teñido un tejido podemos, igualmente, tener conocimiento del origen y fecha de la pieza puesto que determinadas materias se dejaron de usar en épocas conocidas, y de otras se conocen las fechas de importación.

A título de ejemplo, podemos citar un dato sobre dos colorantes rojos estudiados en la Universidad de Delft (Amsterdam) en el que se determinó que entre 1.500 y 1.600 , el insecto kermess desapareció de Europa y durante el período 1.450 a 1.550 otro colorante, la madera de Brasil, no se utilizó.

La utilización del insecto cochinilla (coccus cactis) como colorante rojo se ha usado como dato cronológico para aquellos tejidos cuya presencia se ha constatado. Situándolo como posterior al descubrimiento de Amércia, si bien, en estudios posteriores este dato ha sido cuestionado.

Para la identificación de substancias que se han utilizado en el campo textil se ha empleado una técnica hoy en desuso; la espectroscopia infraroja que sirvió para la identificación de colorantes naturales o sintéticos presentes en los tejidos así como otras substancias, adhesivos, colas, ceras, etc. que han aportado datos muy útiles en el conocimiento de las piezas textiles. Al igual que pueden confirmar datos de los que no hemos tenido referencia mediante la bibliografía.

Este es el caso relacionado con substancias utilizadas en época medieval para evitar el desprendimiento de hilos al cortar una tela. 
La bibliografía suministraba el dato siguiente: «En épocas antiguas no se conocía el sobrehilado de los bordes para evitar que estos se deshicieran, y en su lugar aplicaban cera virgen que al solidificarse evitaba el desprendimiento de hilos». El análisis por espectroscopia infraroja de la substancia de la que hablamos y de la cera virgen confirmó la identidad de los dos elementos.

En determinados casos, puede sernos muy útil recurrir, dentro de las técnicas analíticas no destructivas, a la radiografía. Especialmente en aquellos tejidos en los que el hilo metálico ha sido utilizado bien en la decoración, casi siempre en bordados, o bien en la estructura del propio tejido como elemento enriquecedor del mismo. La ayuda de esta técnica puede ser muy valiosa en el caso en que el hilo metálico del anverso de la tela ha desaparecido. Si el reverso ha conservado el metal de los hilos la radiografía permitirá o bien la lectura si es una leyenda caso que existiera, o conocer con exactitud la técnica de ejecución del bordado.

Dentro de los métodos de análisis no destructivos, se encuentra la espectroscopia de fluorescencia de $\mathrm{RX}$ al que se recurre para obtener datos de una pieza textil con elementos metálicos de adorno o que forman parte del propio tejido.

\section{Factores destructivos de los materiales textiles}

Hay numerosos agentes externos que contribuyen al deterioro de las piezas textiles como son los insectos, los microorganismos, la luz, la suciedad, los aerosoles sólidos, la humedad, la sequedad, la temperatura, etc.

La identificación de los deterioros producidos por insectos o microorganismos se realiza, en el caso de los primeros, mediante microscopia óptica examinando el insecto o resto del mismo y en caso de microorganismos realizando cultivos de las colonias para posteriormente pasar a la identificación taxonómica.

Muchas veces, el origen de los deterioros es el producido por el uso, sobre todo en indumentaria y en la mayor parte, la degradación es irreversible como en el caso de los desgarros, rotos, desgastes, manchas, deformaciones, etc.

Por otro lado se debe tener en cuenta el problema de las intervenciones no documentadas, realizadas por lo general con la intención de mejorar su aspecto estético, sin tener en cuenta su futura conservación, al margen de las intervenciones o modificaciones realizadas 
mientras las piezas estaban en uso, y que pueden constituir auténticos documentos históricos.

Los tejidos forman un grupo de materiales extraordinariamente sensibles a la luz, su exposición prolongada llega a alterar la estructura de las fibras hasta su desintegración y a provocar un debilitamiento irreversible de los colores y una pérdida importante de resistencia y flexibilidad del material.

La radicación infraroja; es decir, las radiaciones de larga longitud de onda situadas a la izquierda del espectro visibles: son poco energéticas y por tanto inocuas en la conservación de los tejidos. Sin embargo, la radiación UV cuya longitud de onda se sitúa a la derecha del espectro, más allá del violeta, de longitud de onda corta y mucha energía, es muy perjudicial para los tejidos.

La iluminación en los museos puede ser de dos tipos: natural y artificial. Esta última puede ser emitida por lámparas incandescentes o fluorescentes que emiten radiaciones U.V. también pero su control es más fácil que en la iluminación natural por ser su nivel de iluminación constante. Las incandescentes de filamento de tungsteno no necesitan ningún control al ser insignificante su emisión de U.V. La iluminación natural está totalmente desaconsejada ya que es la principal fuente de rayos U.V.

El problema de la protección de los tejidos de valor histórico y artístico de la acción de la luz es muy complejo y sus exposiciones deben organizarse dentro de unos límites de seguridad partiendo de que la perfecta conservación se haría manteniendo los tejidos en completa oscuridad, solución esta aceptable solo para aquellos tejidos que se guardan almacenados.

En el caso de los tejidos expuestos es indispensable la eliminación de las radiaciones U.V. mediante filtros. Controlar la iluminación artificial reduciendo el nivel de iluminación, excluir la iluminación natural y evitar la exposición de aquellos tejidos de valor excepcional o extremadamente sensibles a la luz, es una tarea ineludible para todos los conservadores de museos.

La temperatura y la humedad son dos parámetros relacionados entre si y que pueden producir serias alteraciones en los materiales orgánicos.

Tanto una Hr, muy alta como una Hr. Muy baja ocasionan grandes problemas a los tejidos. La primera puede cambiar de dimensiones los tejidos, puede igualmente, producir transformaciones químicas, favorecer la aparición de hongos y en el caso de tratarse de tejidos teñidos debilitar y desplazar los colores. Una caída brusca de la humedad 
relativa del $65 \%$ al $25 \%$, por ejemplo provoca una variación sensible en el debilitamiento de los colores.

La humedad relativa muy baja provoca una fragilidad en los tejidos por la deshidratación de los materiales constitutivos, afectando especialmente a los celulósicos.

Los cambios bruscos de humedad y temperatura producen contracciones y dilataciones en las fibras que al repetirse pueden llegar a producir el agotamiento de las mismas.

Junto con la luz, la temperatura y la humedad son factores que pueden desencadenar degradaciones irreversibles como el caso concreto de rotos.

Para la buena conservación de estos materiales, es fundamental mantener unas condiciones ambientales lo más próximas a una $\mathrm{Hr}$. $55 \%$ y una temperatura de $20 \%$, el control de las variaciones climáticas debe hacerse mediante registros mecánicos y recurrir, si fuera necesario, a humidificadores y deshumidificadores, para impedir que la Hr. Baje del $40 \%$ ya que puede aumentar la fragilidad del tejido. Es importante controlar este parámetro por medio de higrómetros o termohigrómetros.

De forma local, cuando se trata de tejidos expuestos en vitrinas y no se dispone de otro sistema, la $\mathrm{Hr}$. se puede controlar mediante la utilización de silica Gel con indicador de sales de Co.

El polvo en si mismo es un elemento destructor de las fibras textiles sobre todo de las celulósicas, contiene alquitranes como resultado de la combustión pirógena de los combustibles y carburantes, hollín procedente de las antiguas calefacciones y partículas de naturaleza diversa. La gran higroscopicidad del polvo contenido en la atmósfera permite la lenta disolución de la mayoría de estas partículas y su posterior fijación sobre las fibras del tejido además de favorecer la aparición de hongos y mohos.

Otro elemento, tan perjudicial como el anterior para las fibras textiles es el constituido por los aerosoles sólidos que contienen partículas de hierro que al contacto con el anhídrido sulfuroso y el agua de la atmósfera, origina la formación de aerosoles líquidos de ácido sulfúrico que supone un peligro inmediato para los textiles.

El control del polvo y demás partículas contenidas en el aire debe realizarse por medio de filtros especiales que retengan las partículas en suspensión y filtros de carbono activo que absorben el anhídrido sulfuroso, y exponiendo las piezas textiles en vitrinas sin utilizar para su fijación elementos de hierro como alfileres o chinchetas. 
La biodegradación en los tejidos la producen los ataques de insectos y microorganismos. Los primeros fácilmente detectables, por ser muy evidentes, aparecen en los materiales de origen proteínicos aunque en ocasiones algunos insectos larvados en la madera de los muebles pueden atacar a las tapicerías de origen celulósico, y los microorganismos solo se detectan por medio de la microscopia y de test específicos.

Los insectos más frecuentes son la polilla común de los vestidos, la polilla negra, etc. En los climas templados los gusanos y sus larvas producen los mayores daños. Las larvas son más dañinas ya que pueden completar su ciclo vital sobre el tejido. En el caso de la lana estas poseen una substancia que les permite digerir la queratina substancia básica de sus fibras cosa que los insectos no pueden realizar. Por lo tanto al efectuar la desinfección debe hacerse a lo largo de todo el ciclo vital del insecto.

El ataque de los microorganismos no es igual en las fibras de origen vegetal en las de origen celulósico. Los hongos de la celulosa son los microorganismos más peligrosos ya que se nutren de esta substancia y en poco tiempo la resistencia de estos tejidos se ve seriamente afectada. En ambientes con alta humedad relativa la aparición de bacterias que atacan la celulosa es frecuente detectándose un fuerte olor y tacto viscoso en los tejidos.

Las fuentes de infección más corrientes las constituyen los mismos tejidos, en particular los celulósicos sobre todo si llevan restos orgánico, manchas de otro origen, colas, almidón, etc.

El mejor preventivo frente al biodeterioro empieza por la higiene, nunca deben almacenarse cerca tejidos limpios con restos de suciedad o portadores de hongos, mohos o cualquier otro agente contaminante. Antes de añadirlo a otras colecciones debe quedar libre de ellos. En cuanto a las barreras frente a estos agentes se ha comprobado que la mortalidad de 10 larvas de polilla en distintos recipientes con tejidos $\mathrm{y}$ pastillas de p-diclorobenceno es la siguiente:

\begin{tabular}{||l|c|c|c||}
\hline \multicolumn{1}{|c|}{ Recipiente } & Tiempo & Larvas muertas & Larvas vivas \\
\hline Armario sin cerrar Herméticamente & 8 días & 1 & 9 \\
\hline Armario cerrado Herméticamente & 8 días & 9 & 1 \\
\hline Bolsa de papel grueso & 10 días & 6 & 4 \\
\hline $\begin{array}{l}\text { Bolsa de papel grueso Herméticamente } \\
\text { cerrada }\end{array}$ & 7 días & 10 & 0 \\
\hline
\end{tabular}


Las bolsas de plástico no se recomiendan porque pueden condensar la humedad al bajar la temperatura y además atraen el polvo.

Entre los métodos químicos de control contamos con dos cuya efectividad dependerá de las condiciones de los lugares de almacenamiento o exposición, el primero de ellos son las substancias aromáticas como tratamiento preventivo, utilizado desde muy antiguo, su acción es más tóxica que repelente. La naftalina y el p-diclorobenceno son las substancias protectoras más eficaces utilizadas a razón de unos 5 gr./metros cúbicos de aire en las vitrinas o cajones. Sus vapores concentrados matan larvas y huevos de las polillas. Se recomienda usarlos en pequeñas bolsas de tela o papel de celulosa transpirable, que permite el paso de los vapores de estas substancias. Pueden producir manchas, por lo que no deben estar en contacto directo con los tejidos.

Los insecticidas y bactericidas constituyen el segundo de los métodos químicos para luchar contra el biodeterioro, su empleo debe hacerse con mucha precaución en los tratamientos de los tejidos de los museos. Los insecticidas tienen el doble inconveniente de ser muy tóxicos para los humanos y su efectividad no es demasiado larga. Lo más recomendable es utilizar estos productos dentro de las vitrinas de exposición, se puede utilizar p-formaldehido en pastillas a razón de 3-4 gr./metros cúbicos de aire.

Los tratamientos con fungicidas tienen el inconveniente de la elección del más adecuado para conseguir los efectos deseados.

\section{Limpieza}

El estado de limpieza de los elementos textiles constituye uno de los puntos esenciales para su buena conservación, pero es muy importante considerar dos aspectos: el puramente estético de las piezas expuestas en museos y la propia conservación de estas, aspecto este último que en el caso de textiles históricos debe considerarse como primordial.

La acción de la suciedad sobre los tejidos antiguos supone un doble problema: por un lado la naturaleza misma de la suciedad que puede llegar a la destrucción del tejido, y por otro, el substrato-materia textil, sobre la que aquella actúa.

$\mathrm{Al}$ margen de las manchas y los tipos de suciedad referidas más arriba, pueden aparecer, en tejidos procedentes de excavaciones arqueológicas, manchas de tierra y depósitos de materia orgánica cuyo origen sea la descomposición del cuerpo humano con el que mantuvo 
contacto. En estos casos, la eliminación de dichos resíduos es primordial, dado que en ambientes con alta $\mathrm{Hr}$. y aire estancado, la aparición de hongos y microorganismos que se alimentan de esa materia orgánica es segura. El resultado nada favorable por que pueden aparecer manchas redondeadas que van desde el color beige al marrón y negro producto de su metabolismo, que a largo plazo puede suponer la destrucción del material textil o producir transformaciones en las fibras muy perjudiciales para su buena conservación.

Las manchas en los tejidos, como se viene insistiendo, constituyen sin duda un gran peligro para su conservación. En principio puede tratarse de substancias solubles fácilmente eliminables pero que con el transcurso del tiempo sufren determinadas transformaciones químicas que pueden llegar a ser insolubles y necesitar un tratamiento muy agresivo para hacerlas desaparecer. Asimismo, al estar depositadas en la superficie de las fibras, pueden llegar a fijarse dentro de las mismas. Se puede concluir que para la buena conservación de las materias textiles es fundamental la eliminación de la suciedad en lo posible, siempre que la consistencia del tejido lo permita.

La eliminación de la suciedad, por lo tanto, es muy importante para la buena conservación de las piezas textiles, además, porque con este tratamiento se pueden recuperar aspectos fundamentales como son el color, el tipo de ligamento, la decoración del tejido, etc., siendo por otro lado el más arriesgado e irreversible de los tratamientos de conservación y restauración.

Distinguiremos tres tipos de limpieza: mecánica, físico química, y la limpieza mecánica es el procedimiento más simple y eficaz para la eliminación de cierta clase de suciedad, partículas sueltas, depósitos sólidos de distinto origen, huevos de insectos, polvo, etc. Se puede realizar mediante espátula o bisturí, en los casos de acúmulos de sustancias sólidas, cepillado ligero con brochas o cepillos de pelo largo y suave controlando la succión y protegiendo el tejido por una malla que impida el deterioro de la pieza. Las materias succionadas se depositan en un filtro especial adaptado al aspirador para, posteriormente y si el caso lo requiere, ser estudiadas. Algunas piezas con solo esta limpieza será suficiente para su buena conservación.

Para la limpieza de hilos metálicos recubiertos de suciedad, que modifica el aspecto cromático de la pieza donde se encuentra, algunos centros utilizan un sistema de ultrasonidos modificado que elimina la presencia de agua como vehículo transmisor de esta energía para evitar tener la pieza sumergida durante mucho tiempo. Se aplica por 
medio de un punzón cuya cabeza ha sido adaptada a las necesidades del método.

De esta manera se consiguen algunas ventajas; es muy controlable pues en cualquier momento se puede detener la intervención, es rápido, no perjudica el alma del hilo metálico, parece ser que los hilos tratados con este sistema se alteran con más lentitud que por otro procedimiento de limpieza mecánica. Asimismo este método solo es recomendable en materiales que conservan cierta solidez, después de la aplicación de ultrasonidos la pieza debe ser lavada con agua desionizada para eliminar los restos de suciedad y detergente. Este método no se ha generalizado debido a su complejidad y escasas garantías, al mismo tiempo que entraña ciertos riesgos para la pieza tratada.

Pero el procedimiento más utilizado y al mismo tiempo el de mejores resultados es el lavado o limpieza en medio acuoso con detergentes sintéticos.

El lavado de un tejido antiguo en el que las fibras y colorantes han sufrido la acción prolongada del aire, del polvo, de la luz y otros agentes igualmente nocivos, no puede concebirse como el lavado de una tela moderna. Para este último caso un detergente con adición de otros productos como blanqueadores ópticos, enzimas, polifosfatos, carboximetilcelulosa, pueden dar excelentes resultados, pero su acción sobre un tejido antiguo, es fácil suponer que no está exento de riesgos. Por esta razón antes de realizar un tratamiento de lavado es necesario tener algunos conocimientos sobre la naturaleza de la materia textil que va a ser lavada, así como los productos que se van a utilizar.

El lavado es un proceso dinámico en el que se produce el traslado de la suciedad depositada en los tejidos al agua por la acción del detergente. El líquido de lavado disuelve la suciedad quedando esta libre en la solución.

Las fibras naturales lana, seda, algodón sumergidas en agua se comportan como polielectrolitos de alto peso molecular, es decir, existen cargas eléctricas en su superficie. Las fibras celulósicas están cargadas negativamente debido a los grupos $\mathrm{OH}$ hidroxilos libres de sus cadenas moleculares, y en presencia de suciedad que contenga igualmente grupos oxidrilos, aparecen cargas de naturaleza electrostáticas llamadas puentes de $\mathrm{H}$, que fijan la suciedad a la tela; esto ocurre en el caso de manchas de naturaleza orgánica como proteínas, grasas, aceites, etc., $\mathrm{y}$ en otros casos con substancias de tipo inorgánico.

La inclusión mecánica de la suciedad es también importante, las cavidades de las fibras de algodón, y la estructura superficial con es- 
camas imbricadas de las fibras de lana, ofrecen puntos de adhesivos adecuados para toda clase de suciedad.

La función de los jabones y detergentes sintéticos como agentes humectantes o tensioactivos, es reducir la tensión superficial del agua haciendo que esta se extienda y moje el tejido; emulsionar aceites y grasas y envolver las partículas de suciedad manteniéndolas en suspensión para que sean arrastradas al enjuagar el tejido, operación que debe repetirse hasta la total eliminación del detergente.

El campo de los detergentes es extremadamente amplio para exponerlo aquí pero hay que señalar algunos datos importantes referidos a ellos:

La molécula de un detergente se compone de una «cabeza» de carácter hidrófilo y de una «cola» de carácter hidrófobo. Una molécula de detergente en la superficie de una gota de agua orientaría las «cabezas» hacia el centro de esta quedando las «colas» libres para arrastrar la suciedad.

Se puede clasificar los detergentes en cuatro grandes grupos:

- Compuestos de anión activo con carga negativa que liberan aniones, como los sulfatos de alquilo.

- Compuestos de anión activo con carga positiva que liberan cationes, como los compuestos de amonio.

- Compuestos de anfóteros conteniendo grupos cargados positiva $\mathrm{y}$ negativamente $\mathrm{y}$

- Compuestos no iónicos.

Teniendo en cuenta que por su constitución, las fibras celulósicas son fácilmente alterables por substancias generadoras de protones, así como las proteínas por aquellas que liberan cargas negativas, en principio, los tejidos de naturaleza celulósica no deben ser tratados con aquellos detergentes que suministran cargas positivas. Un razonamiento similar podemos hacer sobre las materias textiles de naturaleza proteínica, en principio no será conveniente aplicarles un tratamiento de lavado con detergentes del tipo sulfatos sulfonatos.

Lo más razonable pues, en el lavado de tejidos antiguos, será el uso de detergentes no iónicos como son los procedentes de óxido de etileno y los derivados de ésteres poliglicólicos.

La limpieza de tejidos antiguos mediante verdaderos procesos químicos es la limpieza que podemos llamar orgánica frente a la anteriormente expuesta que se realiza en medio acuoso.

Como cualquier tratamiento de limpieza la elección del método viene condicionada por dos factores: uno, la naturaleza de la suciedad y 
otro la naturaleza misma de la pieza. La limpieza orgánica debe realizarse, siempre y cuando los métodos acuosos carezcan de efectividad, puedan degradar el tejido, y en los que la suciedad a eliminar sea de naturaleza orgánica, es decir, grasas, aceites, etc. o en aquellos en que algún elemento presente en la pieza textil se vea afectado por la acción del agua, como en el caso de la presencia de tintes o colorantes no resistentes al medio acuoso.

Los disolventes utilizados con más frecuencia son: los derivados clorados: dicloro, tri, tetra y percloroetileno, siendo este último uno de los más usados. El dicloroetileno es de acción más suave y conviene utilizarlo en el caso de tejidos teñidos.

Este método, no obstante presenta algunos inconvenientes, que mencionaremos a título informativo: los disolventes orgánicos en especial los aromáticos se inflaman fácilmente, aunque los derivados clorados utilizados frecuentemente no son inflamables. Respecto a los primeros presentan una cierta toxicidad que obliga a tomar precauciones durante la realización de los tratamientos.

Para terminar, no queremos dejar de exponer aquí algunas ideas especialmente dirigidas a aquellas personas que de un modo directo se sienten responsables de la restauración del Patrimonio de Arte Textil:

Que la limpieza de un tejido histórico, no consiste en una renovación total de la pieza mediante una limpieza enérgica que, lógicamente, no podría soportar debido al envejecimiento de sus materiales adquirido por su uso y el tiempo.

Que rechazar por principio productos comerciales inicialmente de gran eficacia, pero de efectos secundarios desconocidos ya que el fin primordial al efectuar cualquier intervención sobre un tejido, debe ser su buena conservación, para transmitirlo íntegro a futuras generaciones.

Que una vez determinada la resistencia de las fibras del tejido que se va a tratar, la solidez de sus colores y la posibilidad de que sufra variaciones de tamaño al entrar en contacto con el agua, se elegirá el método de limpieza más adecuado.

Que debe evitarse el empleo de aguas duras que debido a las impurezas que contienen y a las substancias minerales que tiene disueltas puede perjudicar la pieza textil. A veces basta el lavado con agua para eliminar la suciedad, como quedó expuesto anteriormente si es necesario el empleo de detergentes, estos, serán de las características antes expuestas.

Que cuando la fragilidad de las fibras es extremadamente delicada se recomienda el lavado en plano sin llegar a sumergir el tejido, co- 
locando este sobre una superficie inerte con cierta inclinación y pulverizando con agua desionozada, hasta que la suciedad ablande y los restos gruesos se puedan retirar con instrumentos adecuados. El resto de la suciedad se retira con ayuda de secantes. Esta operación se repetirá hasta obtener la mejor limpieza posible sin llegar a perjudicar la pieza.

Que en el caso de poder manejar la pieza pero asimismo su estado de conservación sea delicado, será preciso, antes de sumergirla, protegerla por medio de una malla dispuesta a modo de sandwich para poder facilitar su manejo en la inmersión. Cuando el tejido está muy sucio, serán necesarios varios baños para retirar la suciedad. En los tejidos muy antiguos se recomienda que esta operación sea lo más rápida posible para evitar que las fibras se dilaten demasiado y pierdan la capacidad de volver a su estado anterior. Una vez sumergida la pieza, para ayudar a la eliminación de la suciedad se puede hacer uso de esponjas naturales aplicándolas a modo de tampón sobre el tejido inmerso en agua y detergente. Esta operación se hará por las dos caras, vuelta de la pieza, se puede realizar con ayuda de un rulo o lámina de material flexible e inerte.

Una vez terminado el proceso de lavado y retirados los restos de detergente, se procederá a la eliminación de las fibras sobre una superficie lisa, y comprobación y rectificación, en su caso, de las medidas originales de la pieza.

Que cuando la solidez de los colores o la naturaleza de las manchas o del propio tejido no aconsejen la limpieza en medio acuoso, se deben usar los disolventes orgánicos de cuya acción se ha hablado más arriba. Para la elección del producto siempre se realizarán pruebas de solidez de los colores y de resistencia de las fibras y se aconseja utilizar para el tratamiento el disolvente de composición más débil.

El efecto de los disolventes orgánicos sobre las fibras, no es el mismo que el producido por el agua, no ablandan las fibras de la misma forma, son muy volátiles y por lo tanto el riesgo de deformación es muy reducido. Hay que advertir que su empleo requiere de ciertas precauciones debido a su toxicidad y, en algunos casos, por ser inflamables.

El tratamiento de limpieza con estos productos se puede realizar de forma local tratando las manchas con isopos impregnados de disolvente y retirando los restos de este y la suciedad con ayuda de secantes y mesa de succión que ayuda a evitar la expansión del disolvente sobre el tejido. 
A veces la suciedad acumulada hace necesario la inmersión del tejido en el disolvente, siendo el proceso de eliminación de esta, igual al aplicado en el caso de la limpieza en medio acuoso.

Tanto en el caso de elegir la limpieza en medio acuoso como en el tratamiento con disolventes orgánicos, antes de iniciarlos se debe hacer una limpieza mecánica para retirar la mayor cantidad de suciedad posible y así acortar el tiempo de inmersión de las fibras y sus posibles consecuencias negativas.

En cuanto a la consolidación de las piezas, en líneas generales, se actúa siguiendo los siguientes criterios:

Los soportes se eligen en base a la naturaleza del tejido a tratar, tanto en cuanto a su composición como a su grosor. El algodón por ser la fibra menos higroscópica de las naturales, y por su facilidad de teñido, es uno de los más adecuados seguida del lino y de la seda. La seda solo se emplea en casos excepcionales pues si se trata de reforzar un tejido de esta naturaleza, ambos tejidos resbalan y se cose mal. Asimismo, la seda, tiene una fuerte reactividad frente a la humedad por lo que es muy fácil que se formen bolsas con los cambios de esta última.

Para la tincción de los soportes se emplean colorantes sintéticos de alta resistencia a la luz y a la humedad.

La crepelina de seda se usa como recurso de excelentes resultados en la matización de los tonos, así como en casos de extremada fragilidad del material en donde no es posible la fijación por cosido en cuyo caso se emplea la crepelina a modo de sandwich para la fijación-conservación del tejido tratado.

Técnica de cosido: El más empleado para la consolidación de los tejidos antiguos es el «punto de Bolonia» realizado con hilos lo más finos posible generalmente de seda «organsin 2 cabos», su envejecimiento es similar al de la seda original de los tejidos antiguos y por lo tanto reaccionarán de forma parecida. Ante piezas de distinta naturaleza la elección de los hilos para la consolidación será la menos perjudicial para cada caso.

A pesar de todos los esfuerzos por rescatar, recuperar y sobre todo, conservar el Patrimonio Histórico Textil, creemos que ha quedado de manifiesto la necesidad de crear unas condiciones ideales de exposición y almacenaje para alargar, en lo posible, la vida de estas piezas de indiscutible valor. Por ello y dado que los textiles son extraordinariamente sensibles a los cambios ambientales no se aconseja los traslados frecuentes que puedan afectar la estructura de las fibras que los forman. 
La metodología que hemos expuesto a lo largo de este artículo no ha sufrido variaciones importantes ni resultados espectaculares a lo largo de varias décadas, esto es debido a que desafortunadamente no se ha dedicado el potencial humano ni económico que estas investigaciones requieren.

\section{Referencias}

Beecher, E. R.: La conservación de los tejidos. UNESCO. París (1.969).

Blanxar ty Pedrals, D.: Materias textiles. Barcelona, (1.954).

Hofenk de Graff, J.: L'Analyse des matières colorantes dans les textiles anciens. Boletin del CIETA. Lyon (1.972).

Hours, Magdalaine. La vie mysterieuse des chefs-d'oeuvre. La science au service de l'art. París 1.980. (Musèe du Louvre).

The textile Institute Mancherter. Identificación de materiales textiles. Mnchester. (1.965).

Lemberg, M. F.: Textile Conservation and research. Abegg-Stiftung. Bern (1.988).

Maschelein Kleener, L.: Les Colorantes des Tapisseries Tournesienne du XV siècle. Bouletin de L'Institut Royal du Patrimoine Artistique. Bruselas.

Parra Greco, E.: Análisis químico de los colorantes del Almohadón de Ximénez de Rada. ICRMC (1.995).

Valentín, Nieves y otros: Control integrado de plagas en bienes culturales de países de clima mediterráneo y tropical. XI Congreso de Conservación y Restauración de Bienes Culturales. Castellón (1.996). 